Article

\title{
A New Meta-Heuristics of Optimization with Dynamic Adaptation of Parameters Using Type-2 Fuzzy Logic for Trajectory Control of a Mobile Robot
}

\author{
Camilo Caraveo, Fevrier Valdez and Oscar Castillo * \\ Division of Graduate Studies and Research, Tijuana Institute of Technology, 22414 Tijuana, Mexico; \\ camilogyc@hotmail.com (C.C.); fevrier@tectijuana.mx (F.V.) \\ * Correspondence: ocastillo@tectijuana.mx; Tel.: +52-664-623-6318
}

Received: 4 July 2017; Accepted: 22 July 2017; Published: 26 July 2017

\begin{abstract}
Fuzzy logic is a soft computing technique that has been very successful in recent years when it is used as a complement to improve meta-heuristic optimization. In this paper, we present a new variant of the bio-inspired optimization algorithm based on the self-defense mechanisms of plants in the nature. The optimization algorithm proposed in this work is based on the predator-prey model originally presented by Lotka and Volterra, where two populations interact with each other and the objective is to maintain a balance. The system of predator-prey equations use four variables $(\alpha, \beta, \lambda, \delta)$ and the values of these variables are very important since they are in charge of maintaining a balance between the pair of equations. In this work, we propose the use of Type-2 fuzzy logic for the dynamic adaptation of the variables of the system. This time a fuzzy controller is in charge of finding the optimal values for the model variables, the use of this technique will allow the algorithm to have a higher performance and accuracy in the exploration of the values.
\end{abstract}

Keywords: fuzzy logic; Type-2; controller; self-defense techniques; herbivores; predator-prey model; Jaccard index

\section{Introduction}

In recent years, the use of dynamic adaptation of parameters in metaheuristics of optimization is a strategy that has had a very important impact in the area of bio-inspired algorithms. The results published by the authors who use this adaptation in their proposals show a very significant improvement in their results. The use of fuzzy logic in the bio-inspired algorithms is an intelligent technique that enables the algorithm to make an intelligent search of values for the parameters and in combination with the algorithm select the optimal values for the solution of the problem.

For this paper, a new variant of the optimization algorithm inspired by the mechanisms of self-defense of plants is presented. In [1], the authors publish the proposal of this algorithm applied to benchmark functions using different methods of biological reproduction: clone, graft, and pollination. The main contribution of this paper is the proposal of a new variant of the algorithm using Type- 2 fuzzy logic for the dynamic adjustment of parameters used to optimize the trajectory of a mobile robot, the objective is that the robot can follow a given reference path with a minimum error.

In the literature $[2,3]$, there are many works with dynamic adaptation of parameters using fuzzy logic for example: In $[1,2]$ the authors use the bee colony algorithm using dynamic tuning of parameters to optimize a controller applied to a mobile autonomous robot. In [4], the author performs an improvement of the Ant Colony Algorithm using interval Type-2 fuzzy logic applied to the travel agent problem (TSP). In [5], the authors propose a new variant of the Particle Swarm Algorithm using Fuzzy Logic FL applied to optimize mathematical functions benchmark. The works mentioned are 
some of those found in the literature, as we observe the use of fuzzy controllers can be used for multiple problems, in recent years they are used to improve the performance of the algorithms [6,7].

The main difference in this work is the proposed meta-heuristic that is being used, the control problems were previously studied by other authors using different optimization meta-heuristics such as some of the following algorithms: Ant Colony [4], Bee Colony [1,3,8,9], Genetic Algorithms [10], Particle Swarm, the meta-heuristics mentioned above were used to optimize the robot trajectory in order to test the performance to complex problems, some of these algorithms were able to find acceptable results and were published as evidence and challenge for other authors [11-14].

Our main contribution in this work is the modification of the bio-inspired algorithm in the self-defense mechanisms of plants in nature using fuzzy logic for the dynamic adaptation of values for the variables of the predator-prey model, as mentioned above this algorithm uses the model proposed by Lotka and Volterra as a basis $[15,16]$. In addition, applying this modified algorithm in the design of the fuzzy controllers for an autonomous mobile robot.

\section{Related Work}

A search in the literature was performed with the purpose of finding works published by other authors that are similar to the proposed algorithm and as a result of the search we find the following works. In the area of fuzzy logic and control, we find the following:

In [2,17-19] ant colony optimization with dynamic parameter based on interval Type-2 fuzzy logic systems are presented. The authors use a combination of intelligent techniques to optimize the trajectory of the robot following a given reference, the use of these techniques allowed the algorithm to have a greater efficiency and stability in the exploration of optimal values.

Also in [16], Ant Colony Optimization using a fuzzy system for TSP problems with dynamic parameter adaptation. The author performs a modification of the ant colony algorithm, using a Fuzzy Logic System FLS to dynamically adapt alpha and rho values, applied to the Travelling Salesman Problem (TSP). Other algorithms have also been combined with FLS for example, in [4] the algorithm of differential evolution is modified with FL for the adjustment of values and applied to mathematical functions as a benchmark. Also in [20], an algorithm based on the harmony search was optimized with fuzzy logic and the performance of the algorithm with fuzzy approach was tested in benchmark mathematical functions.

A new variant of the bio-inspired algorithm based on bats is presented by the author, in [21] Interval Type-2 fuzzy logic for dynamic parameter adaptation in the bat algorithm, the author of this work uses interval Type-2 fuzzy logic to adapt some important parameters in the algorithm and is used to optimize a set of benchmark mathematical functions. In [22], the authors state that: the plants are equipped with an array of defense mechanisms to protect themselves against attack by herbivorous insects and microbial pathogens. Some of these defense mechanisms are preexisting, whereas others are only activated upon insect or pathogen invasion.

Induced defense responses entail fitness costs. Therefore, plants possess elaborate regulatory mechanisms that efficiently coordinate the activation of attacker specific defenses so that fitness costs are minimized, while optimal resistance is attained. In [23], the authors of this paper claim that some of the chemical reactions that release plants can be used to combat pest problems in agriculture. This work is a continuation of some previous publications performed by the authors of this proposed algorithm, for more details see [24].

\section{Self-Defense of the Plants}

The coping strategies of plants are techniques that protect the individual from threats, it was recently shown that the plants have different self-defense mechanisms [23] and these techniques are activated when they detect the presence or a predator attack, some self-defense techniques are as follows. See Figure 1. 


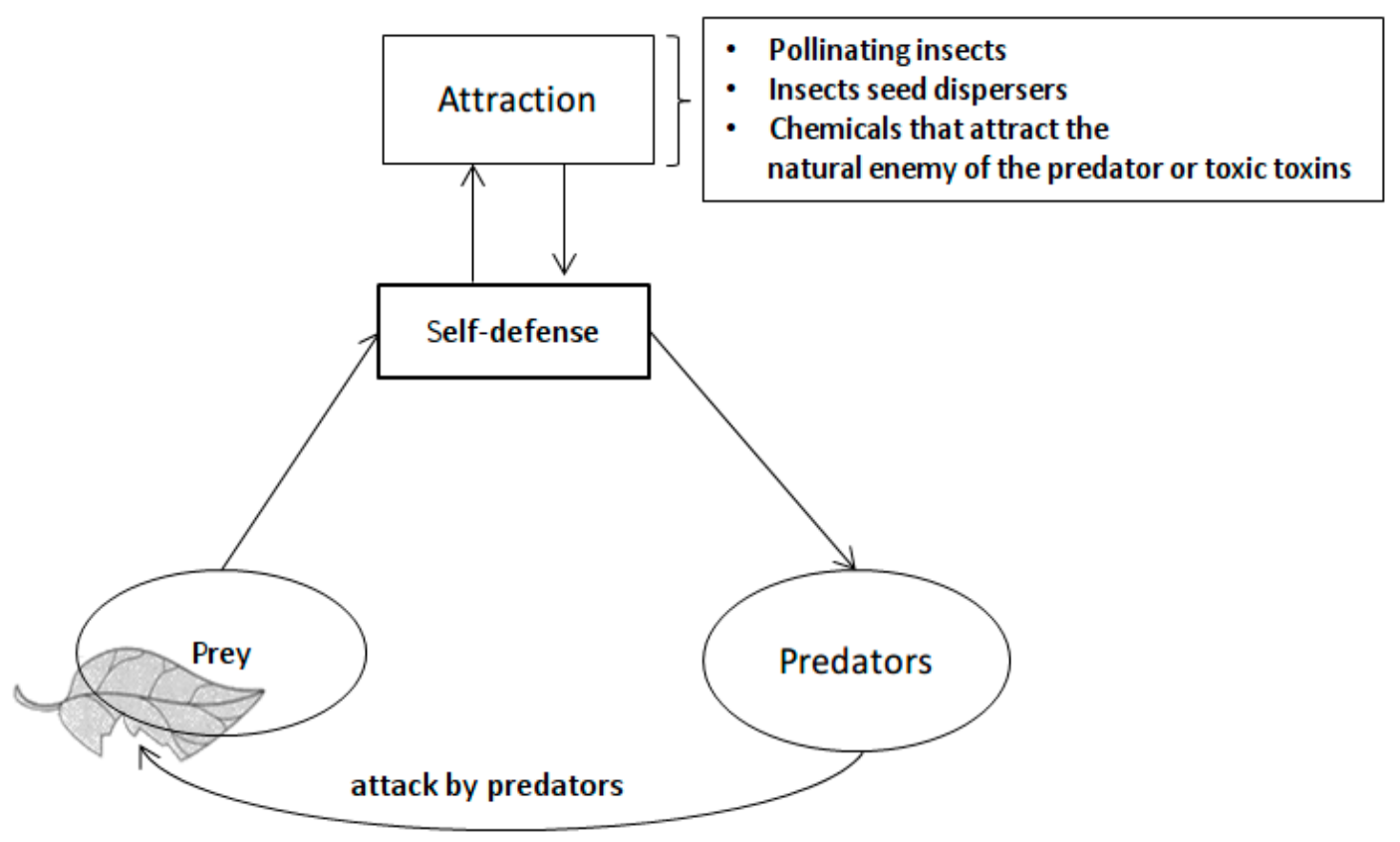

Figure 1. Graphic representation of self-defense techniques.

In $[23,24]$, the authors describe that some of these mechanisms can also be called survival strategies. For example, if the plant is attacked by a predator, the plant can release into the air chemical reactions that attract the natural enemy of that predator, or also activate a mechanism that expels seeds or pollen in the air. For more details see [24]. Plants and all living things have different methods of reproduction for this algorithm, we only consider three clones, pollination and graft, in each method of biological reproduction, a local or global reproduction is determined using probability $\mathrm{p} \in[0,1][24]$.

\section{The Predator Prey Model}

The Lotka-Volterra equations, also known as the predator-prey equations, are a pair of first-order, non-linear, differential equations frequently used to describe the dynamics of biological systems in which two species interact, one as the predator and the other as the prey. The populations change through time according to the following pair of equations [15,16]:

$$
\begin{aligned}
& \frac{d x}{d t}=\alpha x-\beta x y \\
& \frac{d y}{d t}=-\delta x y+\lambda y
\end{aligned}
$$

Equation (1) represents the population growth of plants in the absence of predators. Equation (2) represents the decrease of predator population in the absence of plants at time $t$. where:

$x$ is the number of prey (for example plants).

$y$ is the number of some type of predator (for example, insects).

$\frac{d x}{d t}$ and $\frac{d y}{d t}$ represent the growth rates of the two populations over time.

$t$ represents time.

$\alpha$ measures the birth rate of the plants in the absence of predators.

$\beta$ measures the death rate of the predators in the absence of plants.

$\delta$ represents the susceptibility of plants.

$\lambda$ represents the ability of predation. 


\section{Proposed Method}

In this work, a new meta-heuristic is presented, our proposal is a variant of the algorithm bio-inspired in the self-defense mechanisms of plants in nature with dynamic adaptation of parameters using Type-2 FLS applied to optimize the trajectory of a mobile robot. There is multiple works using Type-2 FLS applied to different optimization problems, the use of this technique significantly improves the results, consult in $[2,5,20,25]$. The principles of Type-2 FLS can be consulted in [17,26-29]. We decided to combine fuzzy logic with our proposal based on works found in the literature, where it is shown that type 2 fuzzy controllers offer a higher performance when applied to robust problems. It is important to mention that in this work, our main contribution is the integration of Type-2 fuzzy logic to the proposed algorithm.

In Figure 2, a flowchart representing the process of the algorithm and we can also observe the stage of the algorithm where we apply Type-2 FL to adjust the values of $(\alpha, \beta, \delta, \lambda)$.

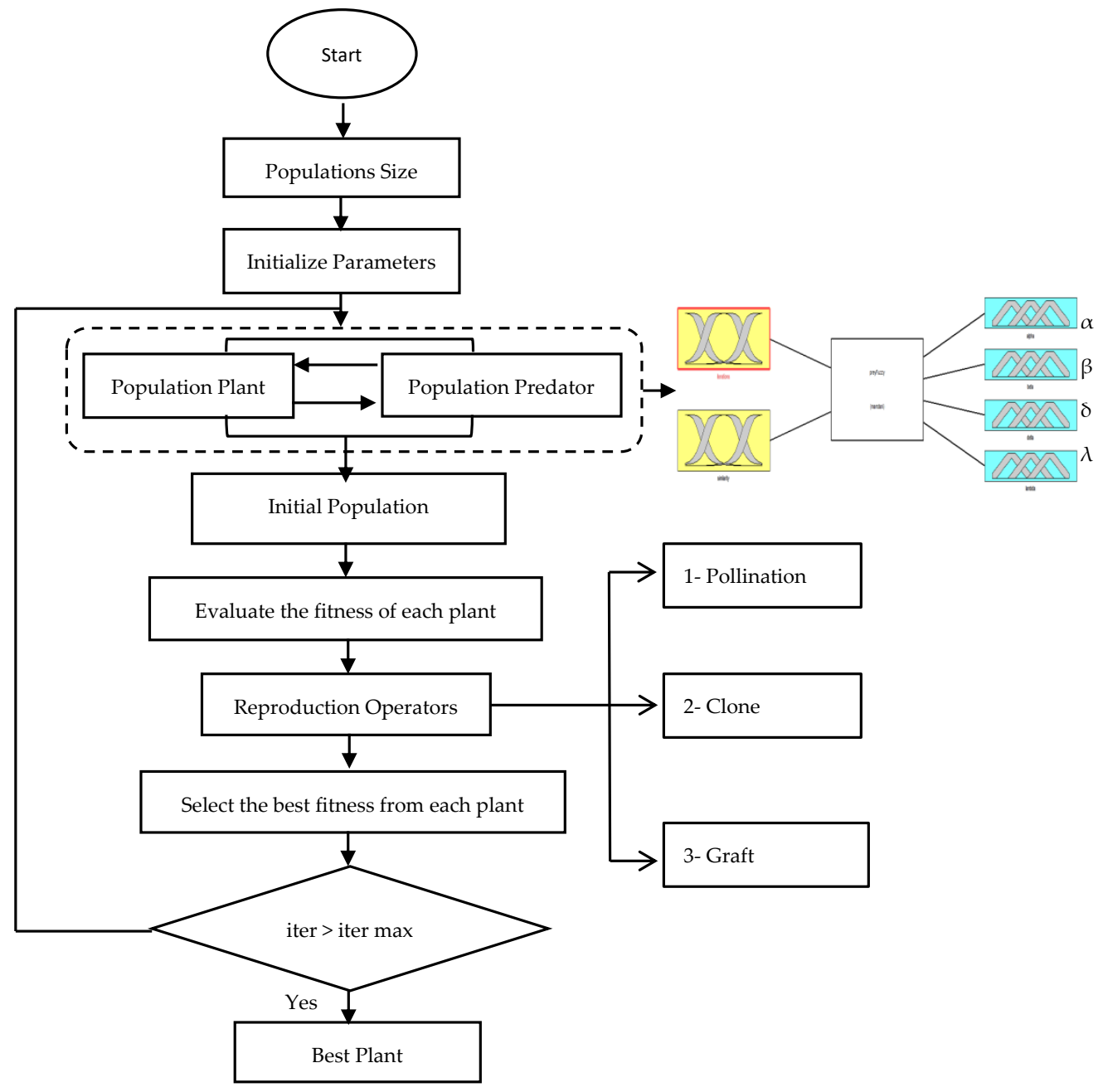

Figure 2. Flowchart with a fuzzy approach.

For this work we are proposing the method of reproduction by pollination, in other previous works using this algorithm with this method of reproduction the results are acceptable.

Pollination: Pollination is a biological reproduction method used by plants to send grains of pollen from one plant (flower) to another plant (flower). In order for this process to be performed, it depends on several factors, in this case the most common are: 
Pollination by insects (biotic): This process of reproduction is totally dependent on birds and insect pollinators, in fact, pollination is more common using bees, when a bee visits a plant to collect honey, it also collects pollen and this pollen is transported to the following plants the bee visits on its way in search for food. This process is also performed by other insects such as butterflies, bats, ants, and other animals [30]. In Figure 3, we can find an illustration of the process of pollination by pollinating insects, where an insect randomly decides to visit the neighboring plant.

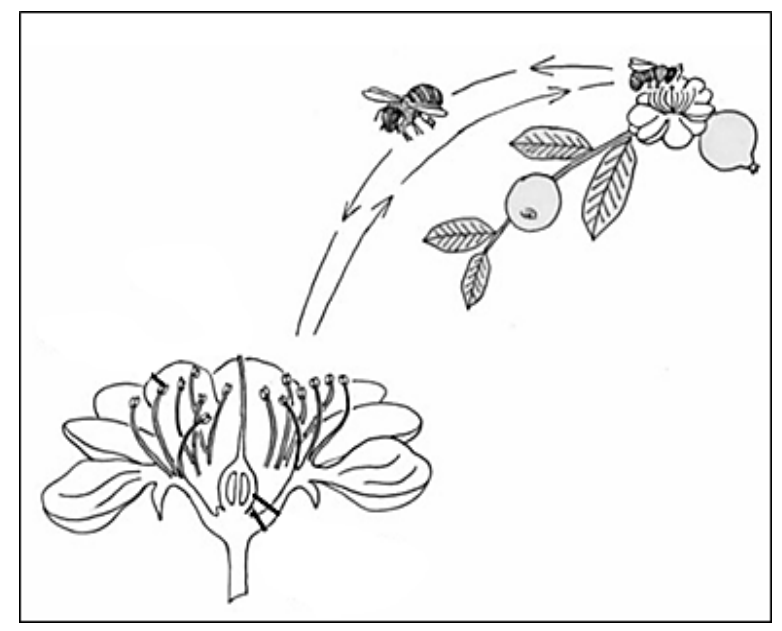

Figure 3. Reproduction method by pollination.

Pollination by air (abiotic). In this case, the pollen produced by plants is transported to other locations using air currents, in this case the air is totally responsible for carrying the pollen from one flower to another flower [30].

The proposed Type-2 FLS controller is of Mamdani Type [31,32], this controller has two variables of input (iteration and diversity) and four variables of output (Alpha, beta, delta, and lambda). In Figures 4 and 5, we can observe the characteristics of the input variables. The variable iteration and diversity is granulated into three membership functions (low, medium, and high) in a range of [0, 1], the type of membership functions proposed for this work are: triangular in the center and trapezoid in the extremes.

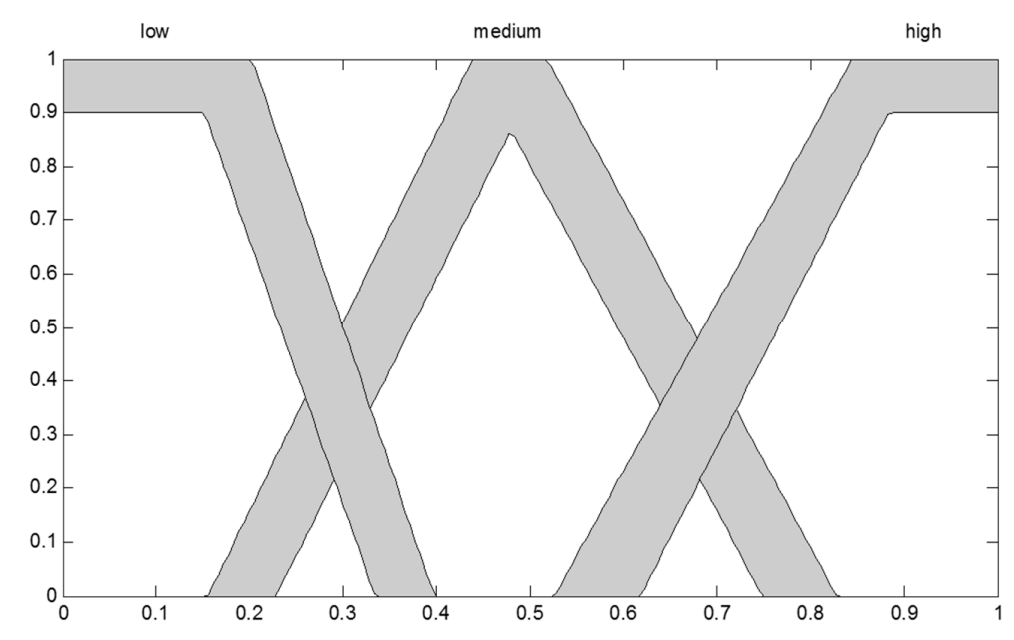

Figure 4. Input variable iteration. 


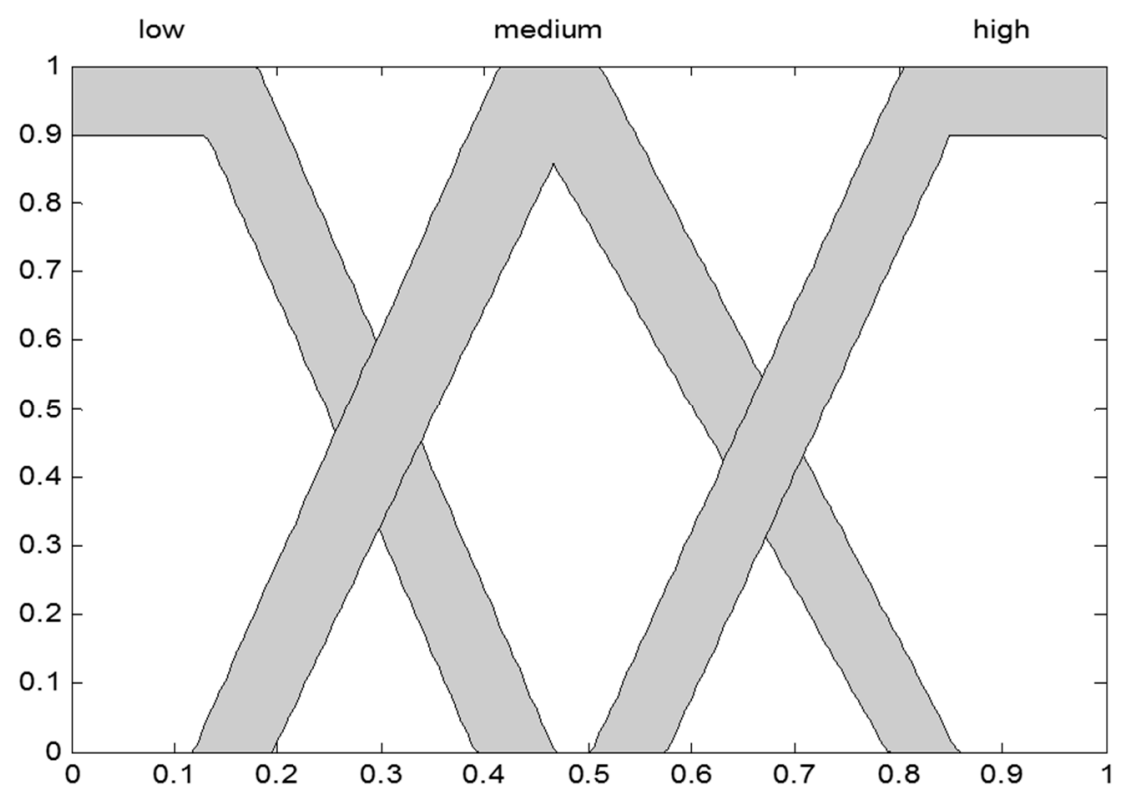

Figure 5. Input variable diversity.

We consider it important to use the iterations value as an input variable of the Type-2 fuzzy controller proposed, using this input variable we can maintain a balance between the exploration and exploitation of the algorithm, because when the iterations are Low we can tell the algorithm to explore and when the iterations are High we can order it to converge in the best region found [5].

The "Iteration" variable is defined by Equation (3), and has a range from 0 to 1; this variable can be viewed as the percentage of the current iterations. In Figure 5, the second input variable diversity is presented.

$$
\text { Iteration }=\frac{\text { Current Iteration }}{\text { Maximun Of Iteration }}
$$

For the second variable input of the proposed fuzzy controller, we are considering the diversity, diversity is a metric that helps us measure how much similarity exists between the population and the best plant found so far. The metric used to measure diversity in this work is the Jaccard index, proposed by Paul Jaccard, it is a statistic used for comparing the similarity and diversity of sample sets. The Jaccard coefficient measures similarity between finite sample sets, and is defined as the size of the intersection divided by the size of the union of the sample sets. The mathematical representation is shown in Equation (4) [33-35].

$$
J(X, Y)=\frac{|X \cap Y|}{|X \cup Y|}
$$

where

1. Count the number of members which are similar between both populations.

2. Count the total number of members in both populations. (Similar and no-similar).

3. Divide the number of similar members (1) by the total number of members (2).

4. Multiply the number you found in (3) by 100.

This metric has also been used by other authors in their works, for more details consult in [32-35]. In Figures 6-9 the four output variables are shown. 


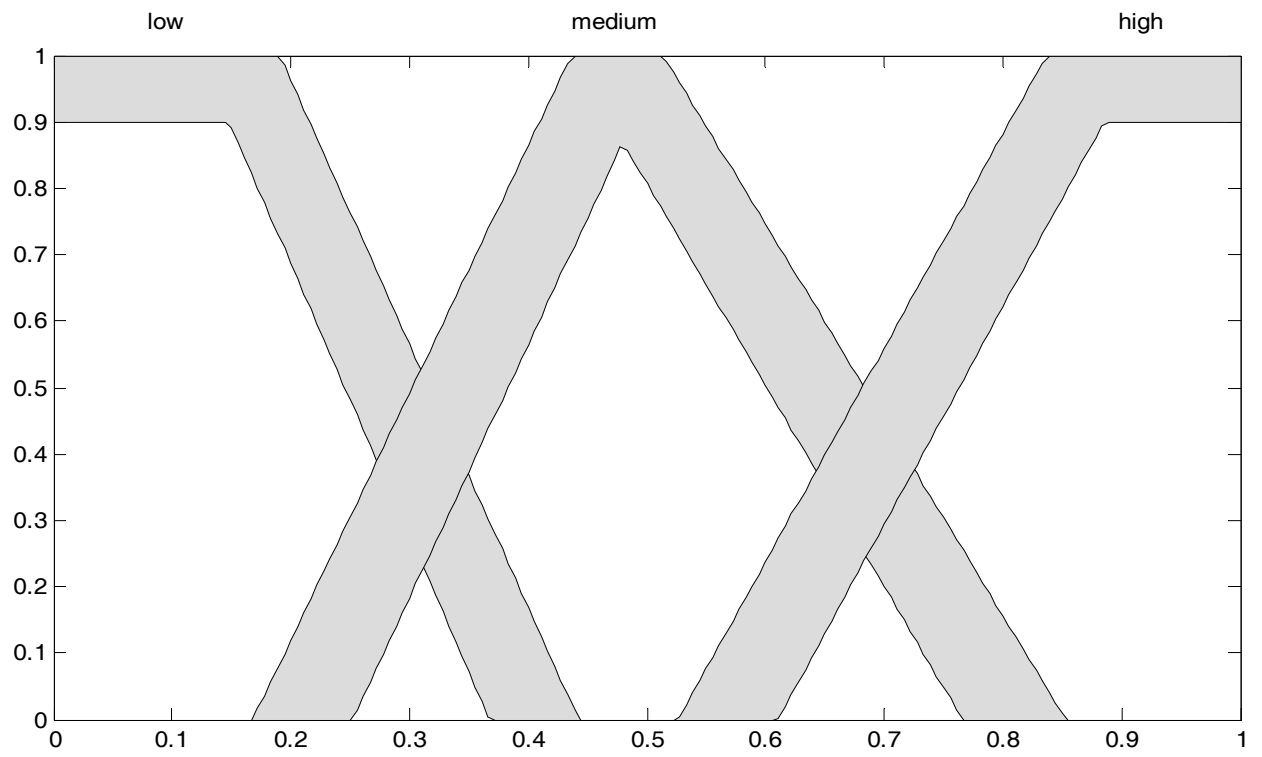

Figure 6. Output 1 Alpha.

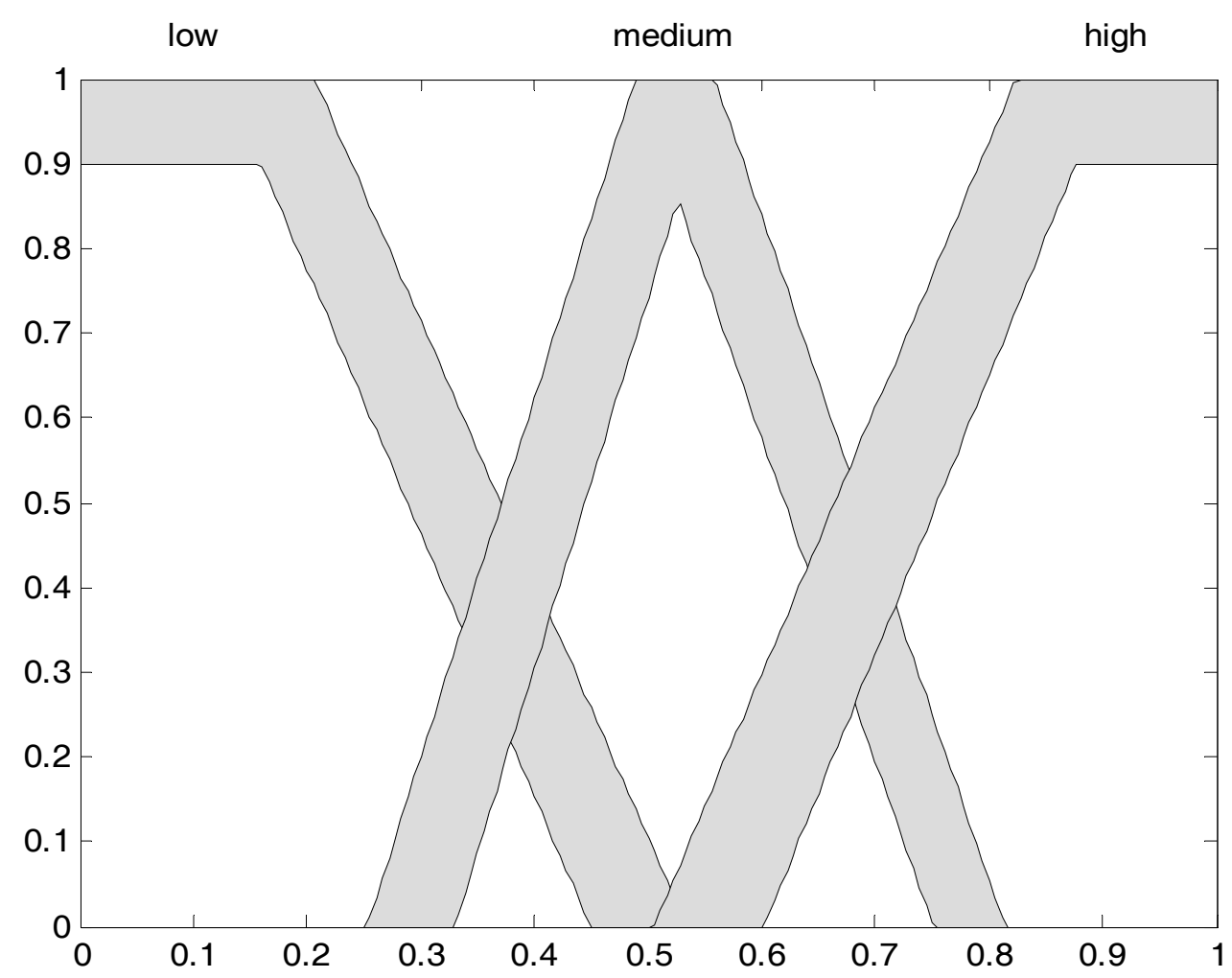

Figure 7. Output 2 Beta. 


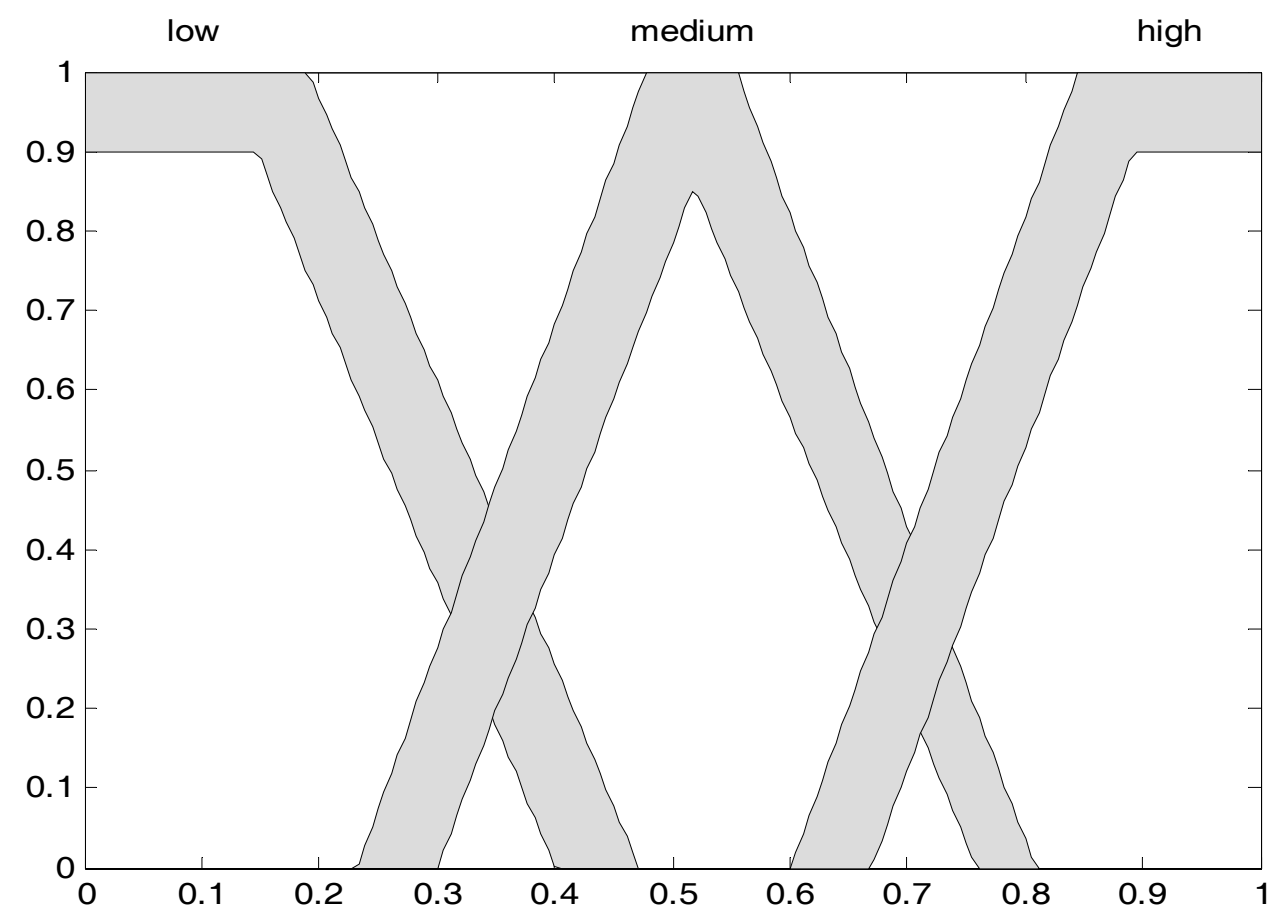

Figure 8. Output 3 Delta.

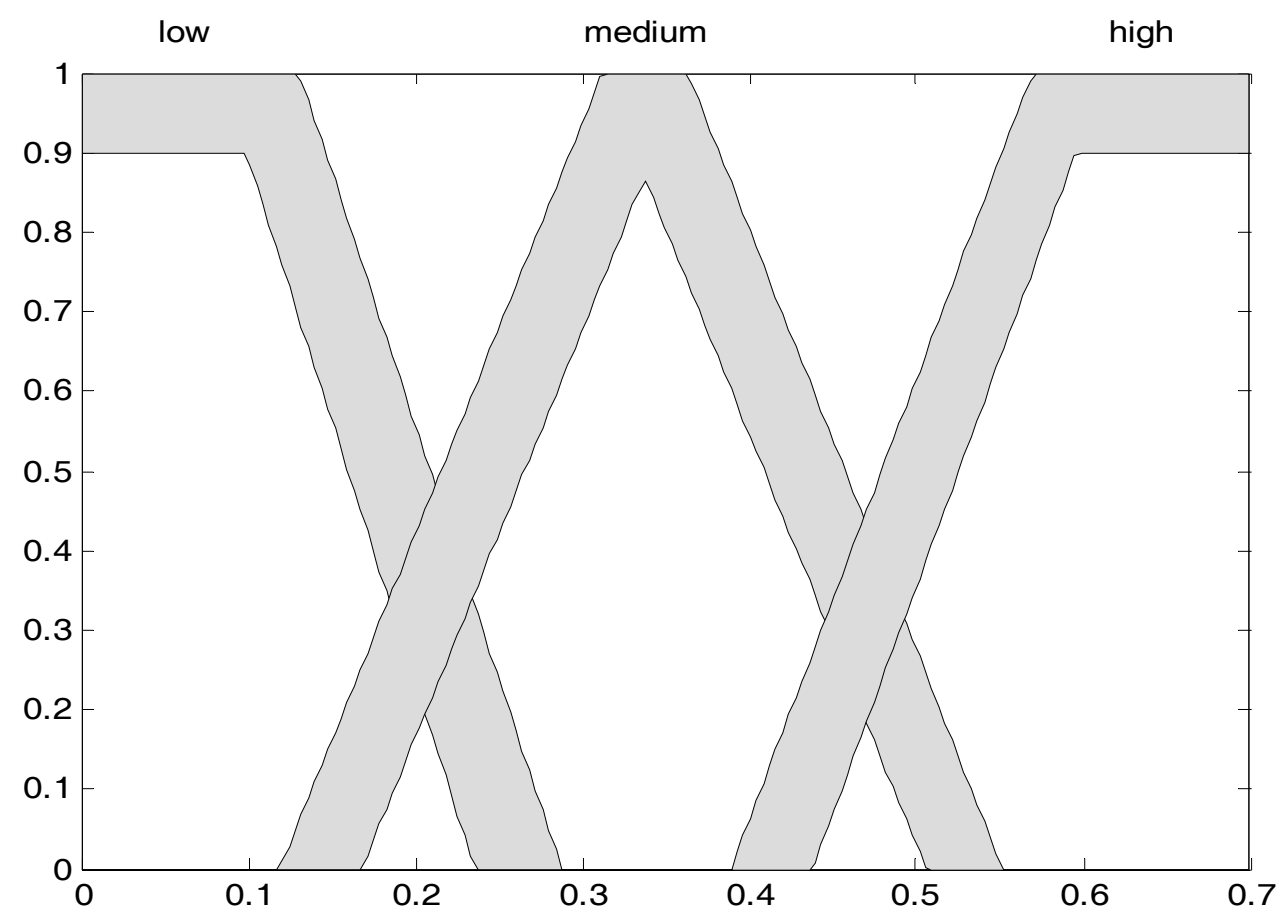

Figure 9. Output 4 Lambda.

The output variables are granulated in three membership functions (Low, Medium, and High) with a range of $[0,1]$, with membership functions triangular in the center and trapezoid in the extremes, and all variables have the same configuration. It is important to mention that these configurations were selected based on prior knowledge of experiments performed and this configuration was the one that gave the best result. 


\subsection{Problem to Be Optimized}

The case selected to apply the proposed algorithm with Type-2 fuzzy approach is the problem of the autonomous robot $[1,5,8,29,36]$, the dynamics of this problem is that the robot is able to follow an assigned trajectory with a minimum error value. In the proposed method an FLS is used to move the parameters of the membership functions of the fuzzy controller. The description of the problem and the characteristics of the fuzzy controller are shown below.

Is an autonomous vehicle capable of following predictable paths in uncertain environments and is illustrated in Figure 10. The robot body is symmetrical around the perpendicular axis and the center of mass is at the geometric center of the body. It has two driving wheels that are fixed to the axis that passes through the center of mass " $C$ " represented by $\left\{C, X_{m}, Y_{m}\right\}$, and one passive wheel that prevents the robot from tipping over as it moves on a plane $[26,27]$. The dynamics of the mobile robot is represented by the following set of Equations (6) and (7), [5,29,36].

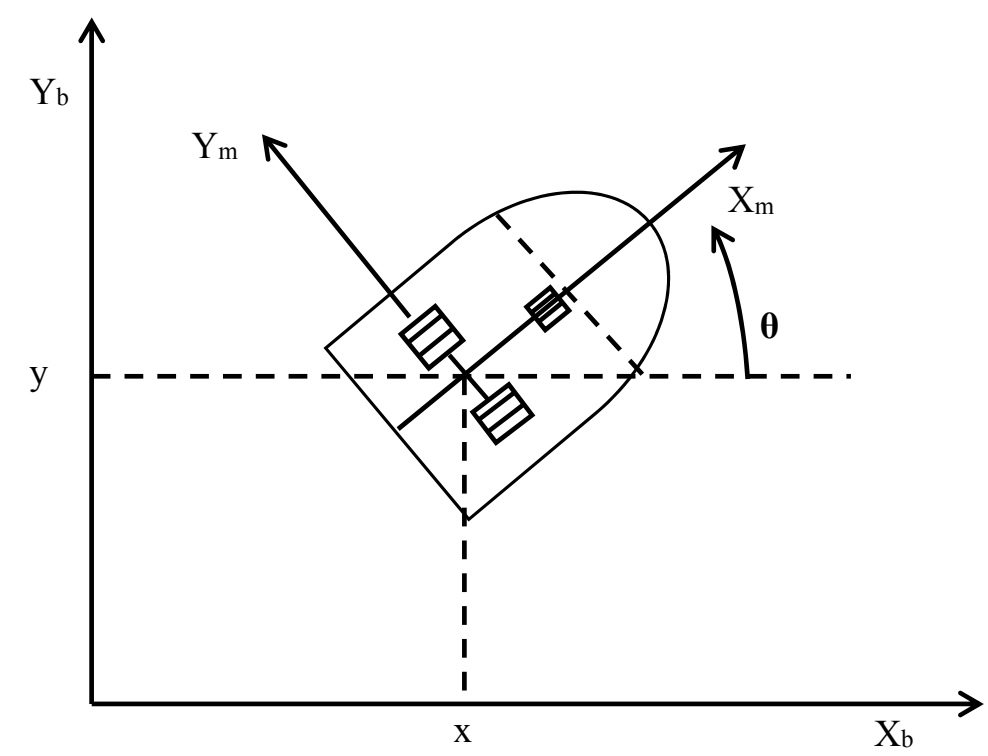

Figure 10. Representation of the mobile robot.

$$
M(q) \dot{v}+C(q, \dot{q}) v+D v=\tau+P(t)
$$

where

$q=(x, y, \theta)^{T}$ is the vector of the configuration coordinates,

$v=(v, w)^{T}$ is the vector of velocities,

$\tau=\left(\tau_{1}, \tau_{2}\right)$ is the vector of torques applied to the wheels of the robot where $\tau_{1}$ and $\tau_{2}$ denote the torques of the right and left wheel,

$P \in R^{2}$ is the uniformly bounded disturbance vector,

$M(q) \in R^{2 \times 2}$ is the positive-definite inertia matrix,

$C(q, \dot{q}) \vartheta$ is the vector of centripetal and Coriolis forces, and

$D \in R^{2 \times 2}$ is a diagonal positive-definite damping matrix.

The kinematic system is represented by Equation (6)

$$
\dot{q}=\underbrace{\left[\begin{array}{cc}
\cos \theta & 0 \\
\sin \theta & 0 \\
0 & 1
\end{array}\right]}_{J(q)} \underbrace{\left[\begin{array}{c}
v \\
w
\end{array}\right]}_{v}
$$


where

$(x, y)$ is the position in the $\mathrm{X}-\mathrm{Y}$ (world) reference frame,

$\theta$ is the angle between the heading direction and the $x$-axis, and

$v$ and $w$ are the linear and angular velocities.

Furthermore Equation (7) shows the non-holonomic constraint, which this system has, which corresponds to a no-slip wheel condition preventing the robot from moving sideways.

$$
\dot{y} \cos \theta-\dot{x} \sin \theta=0
$$

The system fails to meet Brockett's necessary condition for feedback stabilization, which implies that no continuous static state-feedback controller exists that can stabilize the closed-loop system around the equilibrium point.

\subsection{Characteristics of the Fuzzy Controller Used for the Robot}

Below the characteristics of the fuzzy controller used in this case study are presented. We chose to optimize a fuzzy controller of the path for a mobile unicycle robot to test the proposed method in a more complex problem. The controller is of Mamdani type, so that the input and output parameters are represented by linguistic variables. The input variables are the error in the linear velocity $\left(\mathrm{e}_{\mathrm{v}}\right)$ and angular velocity $\left(\mathrm{e}_{\mathrm{w}}\right)$, and the output variables are the right (T1) and left $(\mathrm{T} 2)$ torques $[9,10]$, which are represented in Figure 11.

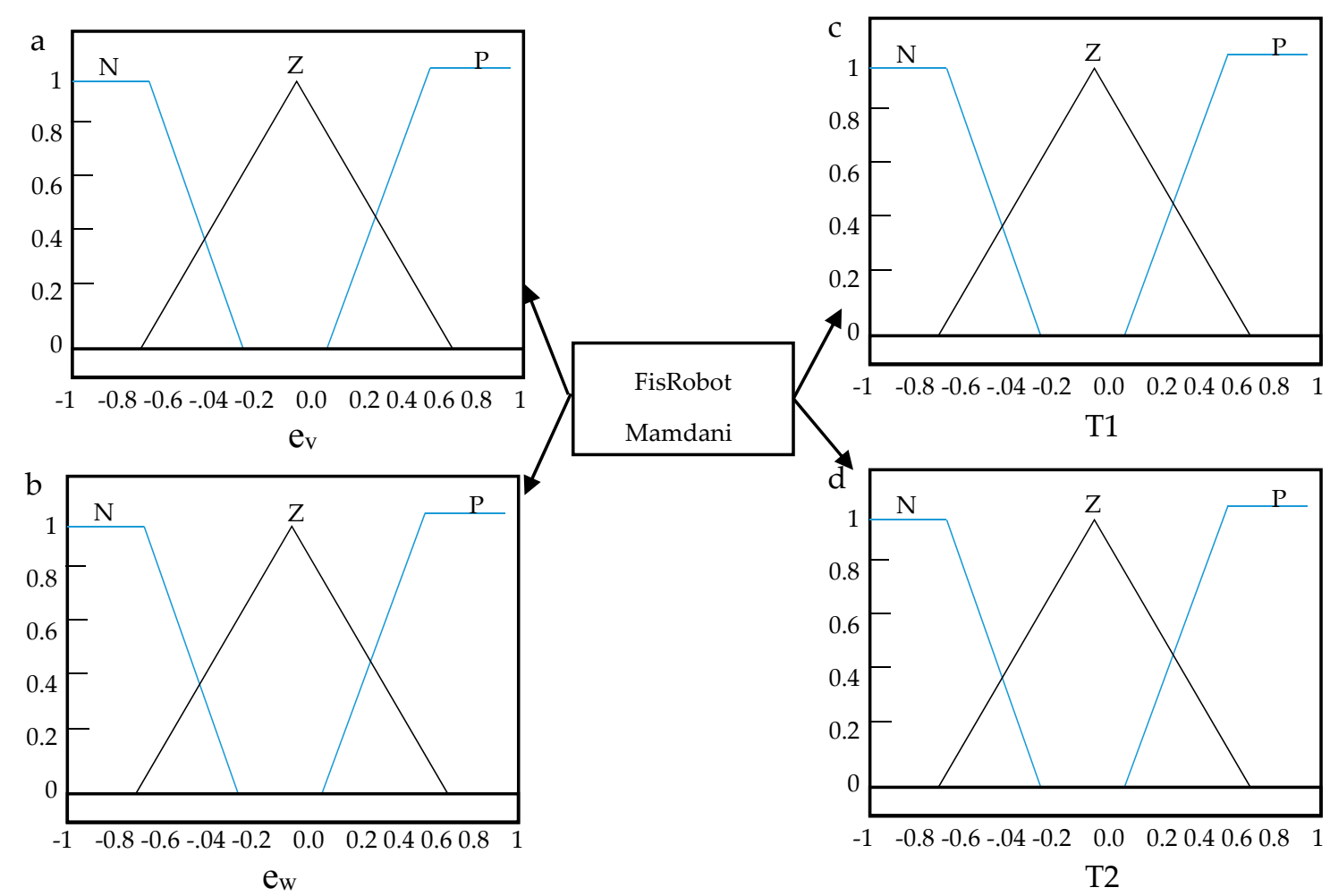

Figure 11. (a) Lineal velocity error $\left(\mathrm{e}_{\mathrm{v}}\right)$; (b) angular velocity error $\left(\mathrm{e}_{\mathrm{W}}\right)$; (c) left torque (T1); (d) right torque (T2).

The membership functions of the input variables are of trapezoidal type in the negative $(\mathrm{N})$ and positive $(\mathrm{P})$ linguistic terms and triangular for the linguistic term for zero (Z). For the output variables we have three membership functions, negative $(\mathrm{N})$, zero $(\mathrm{Z})$, and positive $(\mathrm{P})$ of triangular type. In the range of values of $[-1,1]$ is used for each variable because we have normalized all the variables. 
The fuzzy rules that are used are shown below

1. If ( $\mathrm{e}_{\mathrm{v}}$ is $\left.\mathrm{N}\right)$ and ( $\mathrm{e}_{\mathrm{w}}$ is $\left.\mathrm{N}\right)$ then (T1 is $\left.\mathrm{N}\right)(\mathrm{T} 2$ is $\mathrm{N})$.

2. If ( $\mathrm{e}_{\mathrm{v}}$ is $\left.\mathrm{N}\right)$ and ( $\mathrm{e}_{\mathrm{w}}$ is $\left.\mathrm{Z}\right)$ then (T1 is $\left.\mathrm{N}\right)(\mathrm{T} 2$ is $\mathrm{Z})$.

3. If ( $e_{\mathrm{v}}$ is $\left.\mathrm{N}\right)$ and ( $\mathrm{e}_{\mathrm{w}}$ is $\left.\mathrm{P}\right)$ then (T1 is $\left.\mathrm{N}\right)(\mathrm{T} 2$ is $\mathrm{P})$.

4. If ( $e_{v}$ is $\left.Z\right)$ and ( $e_{w}$ is $\left.N\right)$ then (T1 is $\left.Z\right)(T 2$ is $N)$.

5. If ( $e_{v}$ is $Z$ ) and ( $e_{w}$ is $Z$ ) then (T1 is $\left.Z\right)(T 2$ is $Z)$.

6. If ( $e_{v}$ is $\left.Z\right)$ and ( $e_{w}$ is $\left.P\right)$ then (T1 is $\left.Z\right)(T 2$ is $P)$.

7. If ( $\mathrm{e}_{\mathrm{v}}$ is $\left.\mathrm{P}\right)$ and ( $\mathrm{e}_{\mathrm{w}}$ is $\left.\mathrm{N}\right)$ then (T1 is $\left.\mathrm{P}\right)(\mathrm{T} 2$ is $\mathrm{N})$.

8. If ( $\left(e_{v}\right.$ is $P$ ) and ( $e_{w}$ is $Z$ ) then (T1 is $\left.P\right)(T 2$ is $Z)$.

9. If $\left(e_{v}\right.$ is $\left.P\right)$ and ( $e_{W}$ is $\left.P\right)$ then (T1 is $\left.P\right)(T 2$ is $P)$.

The trajectory to be traversed by the robot is shown in Figure 12 .

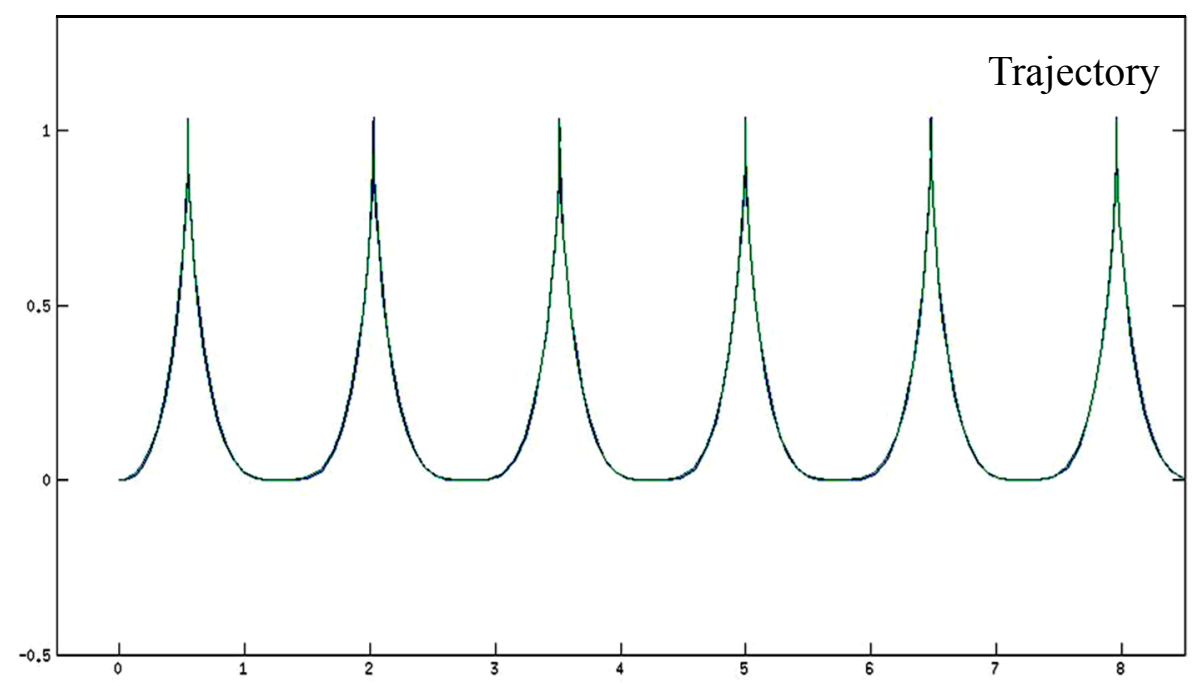

Figure 12. Trajectory to be followed by robot.

The path given to the robot is constant (does not change), and in the course of iterations to the algorithm in combination of Type-2 FLS are responsible for adapting the parameters to direct the robot in that trajectory.

\section{Results Obtained}

The proposed algorithm using Type-2 FLS to adapt the values of the variables of the predatory prey model is used to optimize the trajectory of the problem of the mobile autonomous robot, with the purpose of measuring their performance and stability in complex problems. It is important to emphasize that the proposed algorithm takes as a base the functions of the predator-prey model. In Table 1, we can observe the configuration of parameters used in this work to perform the experiments.

The parameters shown in Table 1 were selected based on previous results and also are used in other works where the same algorithm is used, but applied to a different problem. Also, the results of 30 experiments performed are shown, analyzing the results we observed that the algorithm with Type-2 fuzzy approach has a higher performance and stability, in the values we can observe that they are very similar to the others, that tells us that the algorithm is stable since all experiments are successful, finding acceptable values and we can affirm that the robot is able to follow a trajectory with a greater performance. In Figure 13, the best robot simulation following the reference is shown. 
Table 1. Results obtained and parameters of the algorithm.

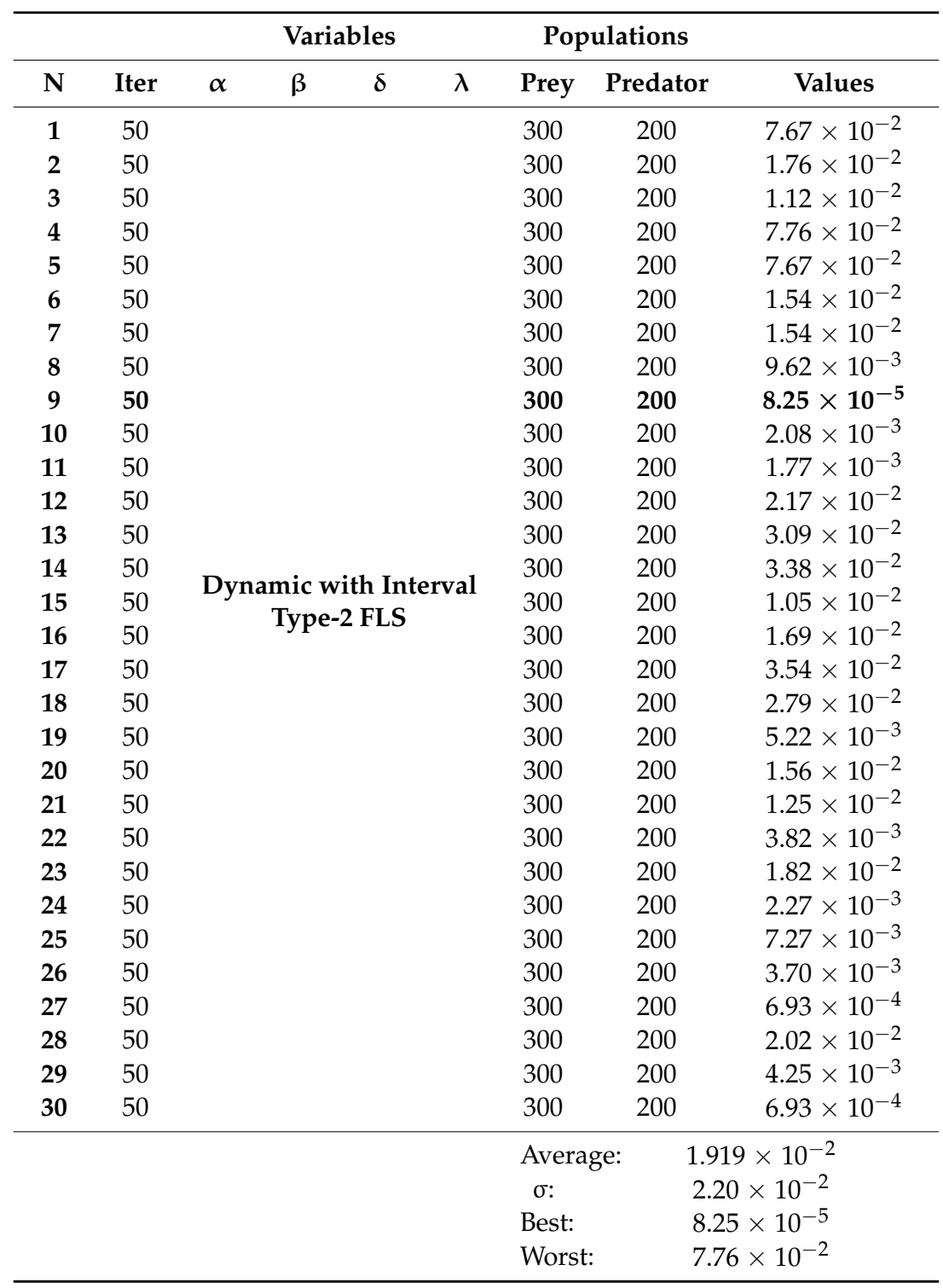

In Figure 11, we can observe a better performance of the fuzzy controller built by the proposed algorithm using Type-2 FLS (see experiment number 9 in Table 1), the blue line represents the reference and the green line represents the trajectory traveled by the robot. The proposed approach has greater stability and adaptation exceeding the expectations of the authors. The errors shown in Table 1 were calculated using the Equation (5) Mean square error [36].

$$
M S E=\frac{1}{N} \sum_{K=1}^{N}[x(k)-y(k)]^{2}
$$

In Equation (5), $x(k)$ represents the actual reference value at time $k, y(k)$ represents the value delivered by the system at time $k$, and $N$ represents the total number of samples considered. 


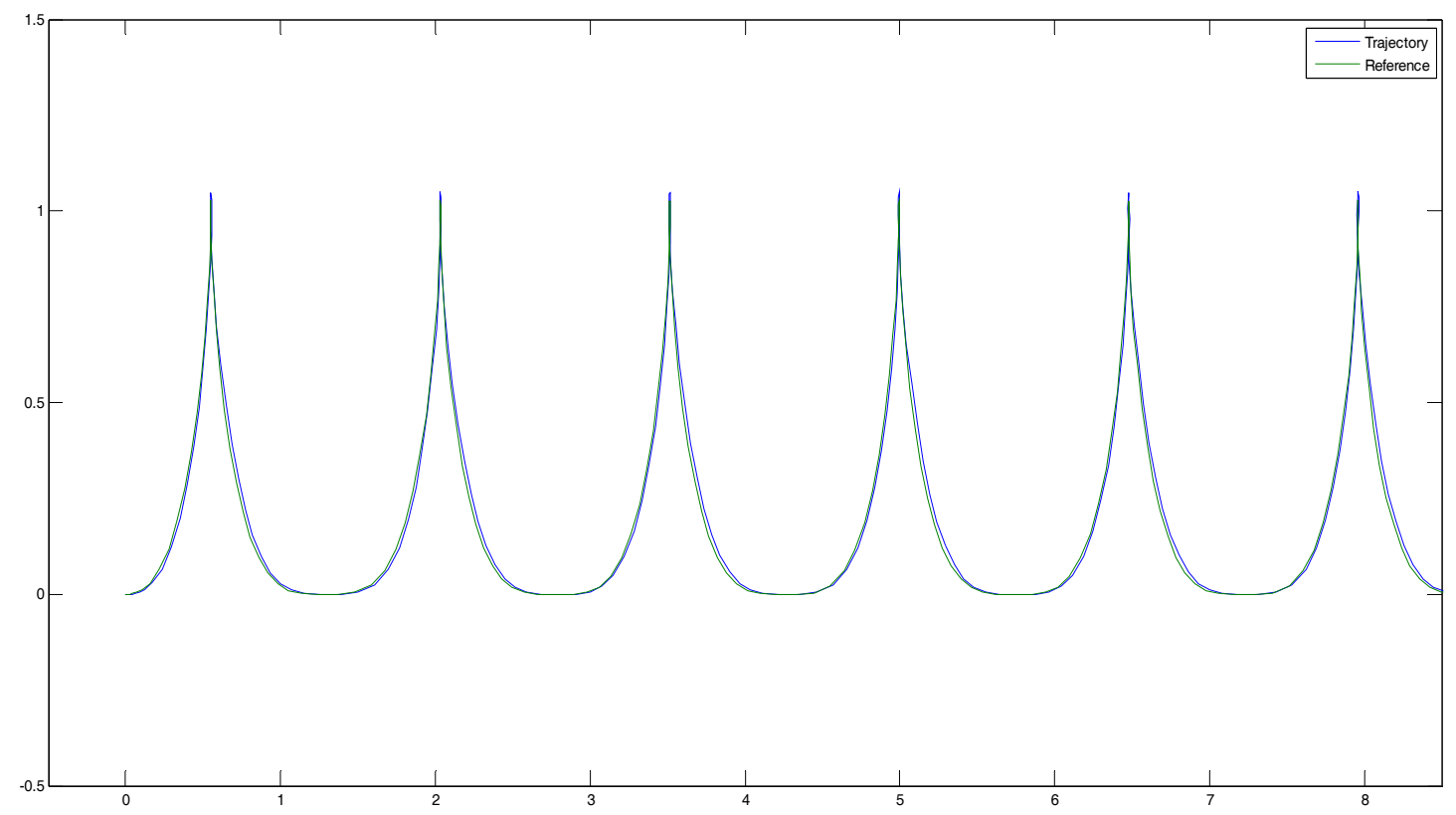

Figure 13. Robot simulation.

\section{Statistical Comparison}

To conclude this work, we consider it important to make a statistical comparison to leave scientific evidence that the algorithm with a Type-2 FLS approach is successful in this case study compared to other works published by other authors and show that our proposal offers a significant contribution. The statistical comparison is made against the Bee Colony Optimization Algorithm with fuzzy logic (FBCO) $[1,9,37]$. The statistical test used for the comparisons is the z-test, whose parameters are defined in Table 2.

Table 2. Statistical z-test parameters.

\begin{tabular}{cc}
\hline Parameters & Values \\
\hline Level of significance & $95 \%$ \\
Alpha & $0.05 \%$ \\
$\mathrm{H}_{\mathrm{a}}$ & $\mu_{1}<\mu_{2}$ \\
$\mathrm{H}_{0}$ & $\mu_{1} \geq \mu_{2}$ \\
Critical Value & -1.645 \\
\hline
\end{tabular}

A significance level of 0.05 is used (that is a usual value), the alternative hypothesis states that the average of the self-defense of the plant algorithm is lower than the average of FBCO, and the null hypothesis states that the average of the proposed algorithm is greater than or equal to the average of FBCO, with a rejection region for all values falling below -1.645 . The statistical test results are that: for the self-defense of the plant algorithm with a fuzzy approach, there is significant evidence to reject the null hypothesis with a calculated value (from the data) of -3.9163 . Clearly this value falls in the rejection region and the alternative hypothesis is true.

Analyzing the statistical test we can affirm that we have sufficient significant evidence to conclude that our proposal is better than the bee colony algorithm with a fuzzy approach, there is also enough statistical evidence to state that in this work we make a successful contribution, and we also observed that the use of FLS as a complement in optimization algorithms significantly improve performance and stability in optimization problems in comparison with other previous works published by the same author of the proposed algorithm [24]. It is also important to mention that the case studies are 
different; in these publications the algorithm is applied to mathematical functions and in this work is applied to problems of fuzzy controller.

\section{Conclusions}

After analyzing the results obtained with the new variant of the algorithm proposed using Type-2 FLS and based on the statistical comparison against the BCO algorithm [37] we have a lot of statistical evidence to say that the self-defense of the plants algorithm with the fuzzy approach, is stable and efficient in this case study. Even though it is a recent algorithm, it proved to be successful in the statistical test; we consider it necessary to test the performance of our new variant in other optimization problems such as: optimize some parameters in neural networks; optimize functions of CEC-2017; optimize other problems in the area of fuzzy controllers just to mention some. We also consider it advisable to use other methods of biological reproduction already integrated in the algorithm, in the case of this study, we only use the method of reproduction pollination using Levy flights, in this work we do not focus on exploring other parameters of the algorithm, since our main objective is to prove that our proposal can be combined with Type-2 FLS to be able to compete against other traditional algorithms and to affirm that although it is recent it is also efficient.

Author Contributions: For Fevrier Valdez contributed to the discussion and analysis of the results; Oscar Castillo reviewed the state of the art, analyzed the data and proposed the method; Camilo Caraveo analyzed the original metaheuristic methods and applied Type-1 and Type-2 fuzzy logic for dynamic parameter adaptation applied for tracking trajectories, and contributed to performing the experiments and wrote the paper.

Conflicts of Interest: The authors declare no conflict of interest.

\section{References}

1. Caraveo, C.; Valdez, F.; Castillo, O. Optimization of fuzzy controller design using a new bee colony algorithm with fuzzy dynamic parameter adaptation. Appl. Soft Comput. 2016, 43, 131-142. [CrossRef]

2. Precup, R.E.; David, R.C.; Petriu, E.M.; Preitl, S.; Rădac, M.B. Novel adaptive charged system search algorithm for optimal tuning of fuzzy controllers. Expert Syst. Appl. 2014, 41, 1168-1175. [CrossRef]

3. Kiran, M.S.; Findık, O. A directed artificial bee colony algorithm. Appl. Soft Comput. 2015, 26, 454-462. [CrossRef]

4. Ochoa, P.; Castillo, O.; Soria, J. Fuzzy differential evolution method with dynamic parameter adaptation using type-2 fuzzy logic. In Proceedings of the 2016 IEEE 8th International Conference on Intelligent Systems (IS), Sofia, Bulgaria, 4-6 September 2016; pp. 113-118.

5. Olivas, F.; Valdez, F.; Castillo, O. Dynamic parameter adaptation in Ant Colony Optimization using a fuzzy system for TSP problems. In Proceedings of the 16th World Congress of the International Fuzzy Systems Association and the 9th Conference of the European Society for Fuzzy Logic and Technology (IFSA-EUSFLAT), Gijon, Spain, 30 June-3 July 2015; pp. 765-770.

6. Zi, B.; Sun, H.; Zhang, D. Design, analysis and control of a winding hybrid-driven cable parallel manipulator. Robot. Comput.-Integr. Manuf. 2017, 48, 196-208. [CrossRef]

7. Zi, B.; Zhu, Z.C.; Du, J.L. Analysis and control of the cable-supporting system including actuator dynamics. Control Eng. Pract. 2011, 19, 491-501. [CrossRef]

8. Amador-Angulo, L.; Castillo, O. Comparative study of bio-inspired algorithms applied in the design of fuzzy controller for the water tank. In Recent Developments and New Direction in Soft-Computing Foundations and Applications; Springer: Cham, Switzerland, 2016; pp. 419-438.

9. Amador-Angulo, L.; Mendoza, O.; Castro, J.R.; Rodríguez-Díaz, A.; Melin, P.; Castillo, O. Fuzzy Sets in Dynamic Adaptation of Parameters of a Bee Colony Optimization for Controlling the Trajectory of an Autonomous Mobile Robot. Sensors 2016, 16, 1458. [CrossRef] [PubMed]

10. Harmanani, H.M.; Drouby, F.; Ghosn, S.B. A parallel genetic algorithm for the open-shop scheduling problem using deterministic and random moves. In Proceedings of the 2009 Spring Simulation Multiconference, San Diego, CA, USA, 22-27 March 2009; Society for Computer Simulation International: San Diego, CA, USA, 2009; p. 30. 
11. Debbarma, S.; Saikia, L.C.; Sinha, N. Solution to automatic generation control problem using firefly algorithm optimized $\mathrm{I}^{\lambda} \mathrm{D}^{\mu}$ controller. ISA Trans. 2014, 53, 358-366. [CrossRef] [PubMed]

12. Yang, X.S. Multiobjective firefly algorithm for continuous optimization. Eng. Comput. 2013, 29, $175-184$. [CrossRef]

13. Yang, X.S.; Hosseini, S.S.S.; Gandomi, A.H. Firefly algorithm for solving non-convex economic dispatch problems with valve loading effect. Appl. Soft Comput. 2012, 12, 1180-1186. [CrossRef]

14. Johanyák, Z.C.; Papp, O. A hybrid algorithm for parameter tuning in fuzzy model identification. Acta Polytech. Hung. 2012, 9, 153-165.

15. Silva, A.; Neves, A.; Costa, E. An empirical comparison of particle swarm and predator prey optimisation. In Irish Conference on Artificial Intelligence and Cognitive Science; Springer: Berlin/Heidelberg, Germany, 2002; pp. 103-110.

16. Xiao, Y.; Chen, L. Modeling and analysis of a predator-prey model with disease in the prey. Math. Biosci. 2001, 171, 59-82. [CrossRef]

17. Barraza, J.; Melin, P.; Valdez, F.; Gonzalez, C.I. Fuzzy FWA with dynamic adaptation of parameters. In Proceedings of the 2016 IEEE Congress on Evolutionary Computation (CEC), Vancouver, Canada, 24-29 July 2016; pp. 4053-4060.

18. Gaxiola, F.; Melin, P.; Valdez, F.; Castro, J.R.; Castillo, O. Optimization of type-2 fuzzy weights in backpropagation learning for neural networks using GAs and PSO. Appl. Soft Comput. 2016, 38, 860-871. [CrossRef]

19. Olivas, F.; Valdez, F.; Castillo, O.; Gonzalez, C.I.; Martinez, G.; Melin, P. Ant colony optimization with dynamic parameter adaptation based on interval type-2 fuzzy logic systems. Appl. Soft Comput. 2017, 53, 74-87. [CrossRef]

20. Peraza, C.; Valdez, F.; Garcia, M.; Melin, P.; Castillo, O. A New Fuzzy Harmony Search Algorithm Using Fuzzy Logic for Dynamic Parameter Adaptation. Algorithms 2016, 9, 69. [CrossRef]

21. Pérez, J.; Valdez, F.; Castillo, O. Modification of the bat algorithm using type-2 fuzzy logic for dynamical parameter adaptation. In Nature-Inspired Design of Hybrid Intelligent Systems; Springer: Cham, Switzerland, 2017; pp. 343-355.

22. Koornneef, A.; Pieterse, C.M. Cross talk in defense signaling. Plant Physiol. 2008, 146, 839-844. [CrossRef] [PubMed]

23. Song, G.C.; Ryu, C.M. Two volatile organic compounds trigger plant self-defense against a bacterial pathogen and a sucking insect in cucumber under open field conditions. Int. J. Mol. Sci. 2013, 14, 9803-9819. [CrossRef] [PubMed]

24. Caraveo, C.; Valdez, F.; Castillo, O.; Melin, P. A new metaheuristic based on the self-defense techniques of the plants in nature. In Proceedings of the 2016 IEEE Symposium Series on Computational Intelligence (SSCI), Athens, Greece, 6-9 December 2016; pp. 1-5.

25. Melin, P.; Castillo, O.; Gonzalez, C.I.; Castro, J.R.; Mendoza, O. General Type-2 fuzzy edge detectors applied to face recognition systems. In Proceedings of the 2016 Annual Conference of the North American Fuzzy Information Processing Society (NAFIPS), El Paso, TX, USA, 31 October-4 November 2016; pp. 1-6.

26. González, C.I.; Castro, J.R.; Martínez, G.E.; Melin, P.; Castillo, O. A new approach based on generalized type-2 fuzzy logic for edge detection. In Proceedings of the 2013 Joint IFSA World Congress and NAFIPS Annual Meeting (IFSA/NAFIPS), Edmonton, AB, Canada, 24-28 June 2013; pp. 424-429.

27. González, C.I.; Melin, P.; Castro, J.R.; Castillo, O.; Mendoza, O. Optimization of interval type-2 fuzzy systems for image edge detection. Appl. Soft Comput. 2016, 47, 631-643. [CrossRef]

28. Perez, J.; Valdez, F.; Castillo, O.; Roeva, O. Bat algorithm with parameter adaptation using interval type-2 fuzzy logic for benchmark mathematical functions. In Proceedings of the 2016 IEEE 8th International Conference on Intelligent Systems (IS), Sofia, Bulgaria, 4-6 September 2016; pp. 120-127.

29. Perez, J.; Valdez, F.; Castillo, O.; Melin, P.; Gonzalez, C.; Martinez, G. Interval type-2 fuzzy logic for dynamic parameter adaptation in the bat algorithm. Soft Comput. 2017, 21, 667-685. [CrossRef]

30. Yang, X.S.; Karamanoglu, M.; He, X. Flower pollination algorithm: A novel approach for multiobjective optimization. Eng. Optim. 2014, 46, 1222-1237. [CrossRef]

31. Singla, J. Comparative study of Mamdani-type and Sugeno-type fuzzy inference systems for diagnosis of diabetes. In Proceedings of the 2015 International Conference on Advances in Computer Engineering and Applications (ICACEA), Ghaziabad, India, 19-20 March 2015; pp. 517-522. 
32. Barbosa, A.M. fuzzySim: Applying fuzzy logic to binary similarity indices in ecology. Methods Ecol. Evol. 2015, 6, 853-858. [CrossRef]

33. Gupta, A.K.; Sardana, N. Significance of clustering coefficient over jaccard index. In Proceedings of the 2015 Eighth International Conference on Contemporary Computing (IC3), Noida, India, 20-22 August 2015; pp. 463-466.

34. Ramli, N.; Mohamad, D. Fuzzy evaluation based on Jaccard with degree of optimism ranking index. In Proceedings of the 2010 International Conference on Science and Social Research (CSSR), Kuala Lumpur, Malaysia, 5-7 December 2010; pp. 970-974.

35. Shi, R.; Ngan, K.N.; Li, S. Jaccard index compensation for object segmentation evaluation. In Proceedings of the 2014 IEEE International Conference on Image Processing (ICIP), Paris, France, 27-30 October 2014; pp. 4457-4461.

36. Melin, P.; Olivas, F.; Castillo, O.; Valdez, F.; Soria, J.; Valdez, M. Optimal design of fuzzy classification systems using PSO with dynamic parameter adaptation through fuzzy logic. Expert Syst. Appl. 2013, 40, 3196-3206. [CrossRef]

37. Teodorović, D. Bee colony optimization (BCO). In Innovations in Swarm Intelligence; Lim, C.P., Jain, L.C., Dehuri, S., Eds.; Springer: Heidelberg/Berlin, Germany, 2009; pp. 39-60.

(c) 2017 by the authors. Licensee MDPI, Basel, Switzerland. This article is an open access article distributed under the terms and conditions of the Creative Commons Attribution (CC BY) license (http:/ / creativecommons.org/licenses/by/4.0/). 